\title{
How Postgraduate Students Use Video to Help Them Learn
}

\author{
Victoria V. Iskru \\ Institute of Psychology and Education, Kazan (Volga region) Federal University, Russia \\ ORCID: 0000-0003-2512-3165 \\ John Schulz \\ Southampton Education School, University of Southampton, Southampton, United Kingdom \\ ORCID: 0000-0003-0007-0749
}

Received: 24 Feb 2020

Accepted: 20 June 2020

\begin{abstract}
Purpose of the study: The object of this paper is to explore the literature in order to discover on what conditions a video-mediated form of instruction can be a learning instrument, the one that enhances teaching, increases the potential of improving learners' performance and leads to a change in students' knowledge and behaviours. Methodology: This exploration is literature based and involves a critical discussion of articles on video-based instruction and video pedagogies. Main findings: Video-based forms of teaching and learning are steadily gaining popularity in the classroom and beyond and are supported by a growing body of academic and professional literature. However, while the pedagogical use of video within a lesson has been explored, what is missing is a focus on the pedagogy within the video - the pedagogical style or design of the video that leads to learning - the transformation of information. Applications of this study: Research involving university students suggests that students conceive of video as an important element of their learning process and they will often choose to use videos for support and clarification rather than any other form of digital instruction. The conducted analysis of the research subject will eliminate concerns of instructional designers over the video design and help employ video more consciously and efficiently. Novelty/Originality of this study: In the last decade, the majority of the literature focusing on creating video for learning purposes adopt a transmission of knowledge approach. Thus, the authors focus on a list of characteristics that will facilitate the transmission of content. Few of the articles focus on learning per se. This paper discusses the results of our review of the literature that support the transmission styles of learning, the "hygiene" principles and then it explores the literature to identify the elements that enable a more transformative learning pedagogy for video.
\end{abstract}

Keywords: video-based instruction, instructional methodology, learning videos, pedagogy, formal learning, digital elements, streaming technology

\section{INTRODUCTION}

Due to the pressure exerted by technology and by the demands of modern technology-savvy learners, tertiary institutions feel the urge to reshape traditional delivery system modalities. Videos are gaining a firm foothold in the educational landscape. It is recognised that videos can season sterile theoretical concepts with live images and sparkle student imagination (Pelton, 2013; Abdous \& Yoshimura, 2010; Chan, 2010). Therefore, teachers in schools and universities are increasingly being urged to adopt video instructional approaches in their teaching and to also instil the skill of learning through video in their students. Empirical studies on the case for using videos report that videos are a powerful medium of presenting educational material, expert demonstrations, giving and revisiting lectures and unfolding complicated scenarios (Berk, 
2009; Moussiades, Kazanidis \& lliopoulou, 2019). Consequently, most universities today have begun to mix live instruction with various digital approaches, which as S.J. Higgins (2003) tells us, is blended or a hybrid learning approach. Video Based Learning (VBL) is an essential component of blended learning especially in 'flipped' classrooms and MOOCs. These days it is hard to imagine modern teaching without the video elements that allow students to engage with the material prior to the class, in their free time. On the other hand, the use of digital elements such as video content is not limited to students undertaking independent study, i.e. to view a specified video in their own time and pace, digital elements are used for formal learning as well. The fact that videos can also be embedded in face-to-face sessions, the use of video is by no means limited to a merely supplementary function (Andrist et al., 2014). However, while contemporary studies consider video as a viable and convenient mode for content delivery, the debate is rarely turned towards viewing video as a mode of teaching that is capable of extending learning, interaction and collaboration opportunities.

\section{PURPOSE AND OBJECTIVES OF THIS PAPER}

Even though present-day technology affordances and access to streaming services make the recording process a far less cumbersome enterprise than ever before and help produce and integrate video with relative ease, it is still not yet understood how to best use videos (Lamb, 2015). The explosion of digital instruction in the past decade has resulted in significant research into using video (Fyfield et al., 2019; Kay, 2012). A cursory review of key findings of the broader literature highlights lines of inquiry such as investigations into video designs, platforms and technologies, and the development of pedagogical approaches. Therefore, the focus of this paper is to explore and identify the principles of video pedagogy in the literature or in other words, the instructional methodology of video. In particular, it is intended to understand how videos can and should be used inside and outside the classroom to good advantage. Furthermore, the study seeks to explore VBL functions for developing students' functional literacy, i.e. critical thinking, creativity, and problem-solving skills.

This exploration is literature based and involves a critical discussion of articles on video-based instruction and video pedagogies. It is also worth mentioning that this paper is a precursor to further inquiry into the potential of VBL to facilitate transformational learning. It is intended to identify elements that enable a more transformational learning pedagogy and to probe the effectiveness of videos. This latter research will analyse students' performance and knowledge acquisition via empirical data and eventually we expect to devise bespoke video-based solutions for teaching in higher educational institutions. Although this paper sets this research within the Russian context, the problem has universal application.

\section{THE RUSSIAN CONTEXT AND RATIONALE}

In their research on eLearning, E.V. Brodovskaya et al. (2019) ponder on the key problems of digitalisation in a number of leading Russian universities. Besides highlighting the depersonalisation of the educational process and lack of some sort of interactive forms of online student support, E.V. Brodovskaya et al. (2019) and her colleagues point out a conspicuous dearth of active digital footprints released by the specified universities. This type of material is generally considered essential to expose and communicate their learning processes to potential students. It becomes more vivid when it concerns these Universities' use of video. The authors insist that Russian universities exhibit a scarcity of digital traces in general, and videos in particular. The unavailability of video resources such as workshops, lectures, presentations, scientific seminars or leading experts' addresses, impact on the pool of potential applicants, the extent of students learning and subsequent career trajectory of graduates.

The number of tutorials or training cases using video is indeed distinctly limited on university webpages in Russia. This indicates, albeit indirectly, the ineffective implementation of practice-oriented tasks into the educational process. Leading Russian higher educational institutions offer video lessons rather thriftily. Less than a third (26.9\%) of the analysed sites contain any video-based training sections. For instance, textbooks (frequently uploaded to the university e-library section) are twice as characteristic (80.8\%) than open access 
full-time training videos (42.3\%). In comparison, learning video material posted on foreign universities' websites amounts to $53.3 \%$. The largest range of educational video content and digital traces can be found on the websites of Tokyo University, the University of Hong Kong and the University of British Columbia (Brodovskaya et al., 2019).

Summarising the results of this research, the use of digital technologies oriented towards providing students factual information dominates overwhelmingly over the styles or technologies that foster interaction and communication. Most noticeably, transmission approaches are presented in the universities' online support for the admission process of applicants and student learning. This approach, also referred to as a so-called 'traditional' digital technology, is highly characteristic of Russian universities. More specifically, it is the kind of technology that supports the educational process merely by placing general, depersonalised information and does not take students' individual educational trajectories into account.

\section{VIDEO PEDAGOGY}

This literature suggests that comprehension of the ways in which videos would mediate the cognitive processes of learning is blurred. Our research initiative attempts to frame the debate into a broader context, though, i.e. to go beyond simply recent developments in the sophistication of video design. The case for video as instruction that is capable of harnessing effective learning experiences and by no means simply another conveyer belt for content should be made. It has to be cogent, well-explained and address areas which, perhaps, do not receive the attention they should be receiving. Hence, before jumping on the bandwagon by integrating video into the educational procedure it deems crucial to fully understand what benefit instructional videos bring to the learning process.

As cognitive science has it, images and audio data if given simultaneously complement each other and assist in making sense of the learning material (Leonard, 2015). According to P.J. Guo, J. Kim and R. Robin (2014), video increases learners' cognitive engagement since through pictorial and auditory channels of sensation it can help process information faster than through written text alone. Secondly, while videos obviously provide visualisation and capture real-world events, they also cater for digital micro-learning and teaching strategies. Digital micro L\&T is what is called a just-in-time, on-demand, bite-sized form of information delivery that appears to be a new nature of the way people learn (Mohammed, Wakil \& Nawroly, 2018).

One study (Andrist et al., 2014) with the intent to bring order on the broad range of films and videos stored in the teachers' growing inventory (over 400 clips), puts forward a teaching typology for videos apt for a course in Sociology. First of all, they make a case for using video for learning - saying that the visual cues help Sociology students make sense of theoretical concepts and notions studied in the course. Additionally, the authors underscore the representational power and expressiveness of videos which in turn enhances students' perception and challenges them to think critically. As far as the teachers' job is concerned, the medium aids them in bridging research with practice, facilitates the delivery of the material and it would be fair to say that in so doing video materials promote learning. Yet to achieve improved learning results, there arises the need for 'practical pedagogy', in other words, pedagogy for instructional employment. The authors state, 'a central task for a practical pedagogy, therefore, lies in developing strategies to bring meaning, order, and appropriate use to this rapidly expanding, undifferentiated mass (i.e. the videos)'. L. Andrist et al. (2014) proceed from the premise that certain types of video are better suited for specific learning goals and subsequently have varying didactic strengths. It is suggested that the typology of this sort, viz. linking video types to learning goals, could lead instructional designers and teachers into thinking about content with special care and deliberation. Consequently, it is hard to escape the conclusion that once videos are conceptually differentiated, it is possible to link their unique properties to mutually exclusive but not collectively exhaustive learning goals.

Pedagogy, no doubt, should assist teaching. Yet, simply stamping videos as educational is hardly enough to advance learning goals. A new typology would be useful as long as it probes somewhat deeper into VBL compared with the commonly accepted typologies based on in-built video features, e.g. animation, interactivity or a 360-degree design, to name just a few. Although L. Andrist et al. (2014) and his colleagues' 
teaching typology might not fit in tightly with a study course other than Sociology, it does initiate discussion on a viable video pedagogy and highlights the fact that without the content being framed in a nuanced and coherent manner, whatever instruments in the teaching toolkit there were they are likely to remain underrated at best or misused at worst.

Despite the recent profusion of video into the T\&L landscape and the observed advantages it offers, there exist certain insecurities about the use of video instruction. And in the first place, instructional designers' express concerns over the video design pedagogy. They do not seem to know how to take advantage of videobased qualities. Instructors need guidance as to how video should be employed for learning in a more consistent and efficient way. Otherwise, either the purpose gets lost on a selected/created video product, or visual cues end up overloading the learner's cognition without ultimately resulting in any sort of maximised learning results (Gašević et al., 2017; Mayer, 2008).

C. Ou et al. (2016) and colleagues put forward a designer approach to video lessons. Drawing the method from instructional design theories they implement their model in a course in artificial intelligence and then conduct a survey on students' perceptions of its effectiveness. The model comes with four pedagogical strategies (learning by example; learning by doing, adaptive learning, i.e. ensuring tutor's personalised feedback; learning through reflection), four instructional phases (activation, demonstration, application, integration), and four guidelines on producing video lesson (limited length; using illustrative charts and tables; conversational format; learner control). The idea behind this research initiative was to leverage the strength of content-based video by following a coherent and dynamic strategy. At this point, the proposed model deems beneficial for instructors and instructional designers as it strengthens video as a teaching medium. However, despite considering certain strategies it does not target learning per se. It does not say whether a persisting change in students' performance or their performance potential come about as a result of this video experience. Nonetheless, the researchers intend to explore further since they clearly draw a fine line between the process of learning and the one of teaching. After all, the authors do not just focus on transmission tools but also the importance of employing the transformational tools pointed out by D.A. Bligh (1998), namely reflections, examples, applications and others. That is to say, besides the students' appreciation of the course and recognition of the instructors' efforts, the subsequent study wants to explore the students' performance in the course which could directly indicate the effectiveness of such a framework.

In their editorial, M. Fyfield et al. (2019) make an overview of key findings of the articles on VBL presented in the Australian Journal of Educational Technology and define video-based pedagogies as 'ways in which videos can be integrated into new teaching and learning practices that take advantage of their affordances such as flexibility, learner control, and the ability to show real-world phenomena in conceptually accessible formats'. Flipped and blended forms of learning are video-based pedagogies. There is no arguing that. Yet, within this research framework we would rather claim that blended learning and its flipped model embodiment are two distinct but at the same time not widely separate types of pedagogy. More precisely, they are two related teaching approaches. We then go as far as to say that basically, they are wrappers for content, the outer layer of the learning process. What we seek to explore here (as well as in the subsequent research) are the principles that underpin pedagogy, that lie beneath the veneer of various shapes and sizes of T\&L approaches and ultimately apply to pedagogy within the video itself. To prevent definitional ambiguity or any other kind of professional jargon from understanding VBL clearly, by video pedagogy in the first place we understand strategies that encourage the learner to be actively involved in the learning process and be in control of their learning. As much as this definition suggests that the learner builds their own knowledge, to a no lesser degree, it originates from the premises of constructivism. After all, what is at issue here are not 'hygiene' principles that would support the transmission of knowledge along a predetermined path, but rather the elements that enable more transformational deeper learning.

\section{REFLECTIONS}

We recognise the necessity of a well-prepared didactics on transmission versus transformational videos, in other words, videos supporting teaching versus videos supporting learning. 


\section{DIFFERENCE BETWEEN TEACHING VERSUS LEARNING PROCESSES}

The following elaboration on essential differences between the processes of teaching and the actual act of learning is built upon three points of reference. Point one speaks to the problems of formation of new competencies mandatory for the new Third Industrial Revolution (the term coined by Jeremy Rifkin, an American economic and social theorist). Most notably, the revolution changes the requirements for crosscutting competencies that permeate the entire structure of the labour market. If there are $\mathbf{7 0}$ million people in the present-day labour market, then every tenth should possess these competencies.

To move on to point two, it is crucial to acknowledge that over the past 150 years there has been a fundamental paradigm shift in teaching. A German philosopher, Johann Friedrich Herbart (1776-1841), wrote that the child must be made limp so that he becomes a malleable, receptive vessel fit to absorb wisdom and salvational truths. He went on to say that the student cannot have his own will or goals other than those set by the teacher. Hence, by Herbart, a student is an object of pedagogical influence who takes a response position and does not have the opportunity for his own goal-setting and goal-realization in the learning process. Only 100 years ago, Sergey Hessen said that the assumption that the pedagogical process conveys knowledge is erroneous. The idea is that teachers do not transfer knowledge; they transmit information. Teachers systematise knowledge and transfer the experience of previous generations to a new generation which does not necessarily lead to learning. What the new generation will know is what their predecessors came up with. While knowledge can arise only if the student does something themselves. And in order to do something, they need not information, but a method. Therefore, students need to be taught methods and do everything themselves. This came to be known as a new ontology of the pedagogical process, a new image of the world in the mind of a teacher.

The last point recaps on learning versus teaching. The first step in the development of the new ontology is the acknowledgement that the processes of learning and teaching should be separated and contrasted. There exists such an idea that the pedagogical activity is defined by three components: learning goals, content and methodology (i.e. the organisation of the educational process). The question is whose are the goals and how are they coordinated? Etymologically, the word 'content' implies 'joint holding'. Therefore, it cannot be only at the teacher's disposal. Until this is taken, internalised and appropriated by the second participant, this is not about content. So is the performance of the pedagogical process. It is determined not by the teaching process, but by the learning process. Efficiency can only be in the activity that the student performs in certain conditions. Learning can have a result, can be measured. And the effectiveness depends on what work the student does during the educational process and beyond. What should be regarded as a positive result of pedagogical activity is a person's ability to perform a productive action, specifically when the action the student takes is vigorous, independent, intentional and prudent.

To round it up, the new interpretation of the educational process is only 150 years old. Within this new framework little has been done. Quite so often learning outcomes are the fruit of an unequal contest between the infinite possibilities of a man and the educational system. Yet, it is believed that by raising awareness of new pedagogical strategies, new teaching ontology, it will be possible to transform the process of learning and VBL may have the potential for that.

\section{AN EXAMPLE FROM PRACTICE}

In one of his teacher training sessions on digital learning the co-author of this paper, John Schulz, taught the postgraduate students who major in Education how to create a learning video. Proliferating from a vast body of research literature the tutorial was segmented into the elements found crucial for designing and developing videos. This model is drawn from the work of J. Koumi (2006) and G. Eustace (1990). The layout was as shown in Figure 1. 


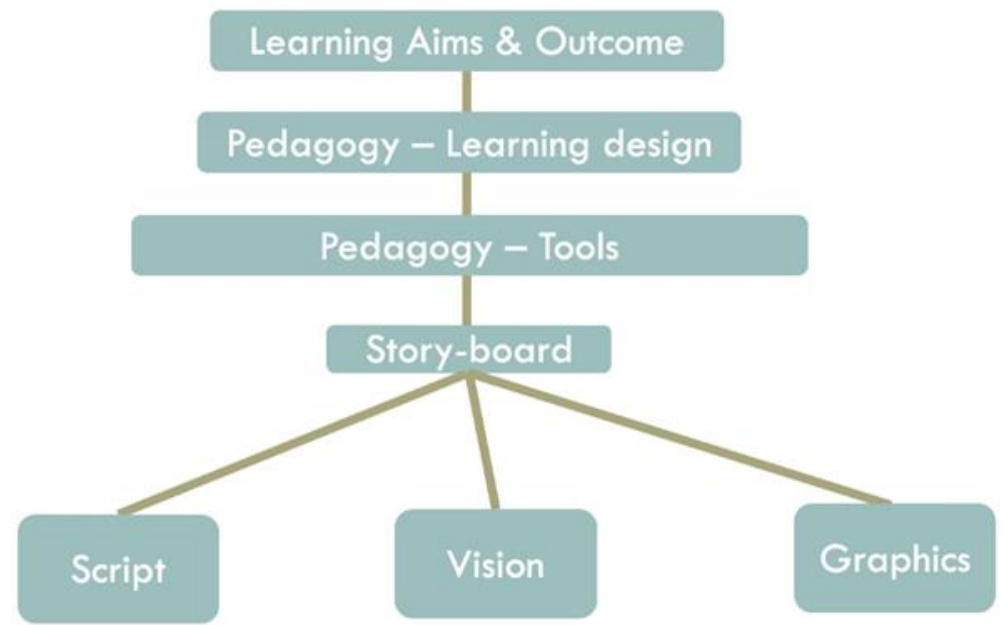

Figure 1. Framework for creation of a learning video

First of all, students were to specify the desired aims and potential learning results of their video. That would also involve considering the context of viewing, the target audience, and the students' prior knowledge. The second step was to have students focus on the design itself, i.e. whether video is designed to transmit certain information to the audience or help them transform it into newly constructed concepts.

This approach to teaching educational video design correlates closely with a fair number of research assumptions and findings in the field of instructional theory. Since the tutorial starts out by defining learning aims and objectives for the to-be-designed video it particularly overlaps with the aforementioned work of $\mathrm{L}$. Andrist et al. (2014). It was believed that raising students' awareness of two distinct pedagogical strategies would lay the groundwork for them thinking of video not only as a teaching medium but a learning tool as well.

\section{CONCLUSION}

Having undertaken the research project which aims to explore through literature the potential of video to help students learn it was made possible to carefully specify what is basically conceived of video learning.

- Video learning is the result of students' cognitive engagement in meaningful learning experiences whereby they interact with the material and learn to construct their own knowledge.

- The student's ability to apply new information to solve a problem in a different but related context is perceived as academic achievement.

- It is essential that video learning should be designed in a way that elicits connectedness of the material to be learned with students' past knowledge and real-life situations.

- Educational philosophy remarkably consistent with this view of teaching would be constructivism. This school of thought emphasises that knowledge is not some designated body supplied in some predetermined way but a construct to be actively built by the student.

- The teacher's role is first, to create conditions whereby students' old information transacts with new information and second, to assist them in discovering their full potential.

- Transformational learning has, in a sense, room for self-actualisation of both students and teachers alike. Although this teaching approach surely requires open-mindedness on the part of the latter. It needs a reevaluation of teacher roles from being the dispenser of knowledge and measuring agency to a fellow student.

- A holistic video educational experience might as well incorporate all the two views of T\&L, transmission and transformational, so long as they add meaning, consciousness and interconnectedness to learning. 


\section{ACKNOWLEDGEMENTS}

The work is performed according to the Russian Government Program of Competitive Growth of Kazan Federal University.

\section{REFERENCES}

Abdous, M. H., \& Yoshimura, M. (2010). Learner outcomes and satisfaction: A comparison of live videostreamed instruction, satellite broadcast instruction, and face-to-face instruction. Computers \& Education, 55(2), 733-741. https://doi.org/10.1016/j.compedu.2010.03.006

Andrist, L., Chepp, V., Dean, P., \& Miller, M. (2014). Towards a Video Pedagogy: A Video Typology with Learning Goals. Teaching Sociology, 42(3), 196. https://doi.org/10.1177/0092055X14524962

Berk, R. A. (2009). Multimedia teaching with video clips: TV, movies, YouTube, and mtvU in the college classroom. International Journal of Technology in Teaching and Learning, 5(1), 1-21.

Bligh, D. A. (1998). What's the Use of Lectures? Intellect books.

Brodovskaya, E. V., Dombrovskaya, A. Yu., Petrova, T. E., Pyrma, R. V., \& Azarov, A. A. (2019). Digital environment of leading universities in the world and the Russian Federation: results of a comparative analysis of these sites. Higher Education in Russia (Vysshee obrazovanie v Rossii), 28(12), 9-22. https://doi.org/10.31992/0869-3617-2019-28-12-9-22

Chan, Y. M. (2010). Video instructions as support for beyond classroom learning. Procedia-Social and Behavioral Sciences, 9, 1313-1318. https://doi.org/10.1016/j.sbspro.2010.12.326

Eustace, G. (1990). Writing for Corporate Video. Oxford: Butterworth-Heinemann.

Fyfield, M., Henderson, M., Heinrich, E., \& Redmond, P. (2019). Videos in higher education: Making the most of a good thing. Australasian Journal of Educational Technology, 35(5), 1-7. https://doi.org/10.14742/ajet.5930

Gašević, D., Mirriahi, N., Dawson, S., \& Joksimović, S. (2017). Effects of instructional conditions and experience on the adoption of a learning tool. Computers in Human Behavior, 67, 207-220. https://doi.org/10.1016/j.chb.2016.10.026

Guo, P. J., Kim, J., \& Robin. R. (2014). How video production affects student engagement: An empirical study of MOOC videos. ACM Conference on Learning at Scale (L@S 2014). https://doi.org/10.1145/2556325.2566239

Higgins, S. J. (2003). Does ICT improve learning and teaching in schools? BERA, British Educational Research Association. https://www.worldcat.org/title/does-ict-improve-learning-and-teaching-in-schools/oclc/ 770684577

Kay, R. H. (2012). Exploring the use of video podcasts in education: A comprehensive review of the literature. Computers in Human Behavior, 28(3), 820-831. https://doi.org/10.1016/j.chb.2012.01.011

Koumi, J. (2006). Designing Video and Multimedia for Open and Flexible Learning. Routledge. https://doi.org/10.4324/9780203966280

Lamb, R. A. (2015). A Makeover for the Captured Lecture: Applying Multimedia Learning Principles to Lecture Video (Doctoral dissertation). Fort Lauderdale: Nova Southeastern University. Retrieved from https://nsuworks.nova.edu/gscis_etd/36

Leonard, E. (2015). Great Expectations: Students and Video in Higher Education. Sage White Paper.

Mayer, R. E. (2008). Applying the science of learning: Evidence-based principles for the design of multimedia instruction. Cognition and Instruction, 19, 177-213. 
Mohammed, G. S., Wakil, K., \& Nawroly, S. S. (2018). The effectiveness of microlearning to improve students' learning ability. International Journal of Educational Research Review, 3(3), 32-38. https://doi.org/10.24331/ijere.415824

Moussiades, L., Kazanidis, I., \& Iliopoulou, A. (2019). A framework for the development of educational video: An empirical approach. Innovations in Education and Teaching International, 56(2), 217-228. https://doi.org/10.1080/14703297.2017.1399809

Ou, C., Goel, A. K., Joyner, D. A., \& Haynes, D. F. (2016). Designing videos with pedagogical strategies: Online students' perceptions of their effectiveness. In Proceedings of the Third (2016) ACM Conference on Learning@Scale, pp. 141-144. https://doi.org/10.1145/2876034.2893391

Pelton, J. A. (2013). "Seeing the Theory Is Believing" Writing about Film to Reduce Theory Anxiety. Teaching Sociology, 41(1), 106-120. https://doi.org/10.1177/0092055X12462142

Correspondence: Victoria V. Iskru, Research Associate, Institute of Psychology and Education, Kazan (Volga Region) Federal University, Kazan, Russia. E-mail: victoria.iskru@gmail.com 